\title{
NOUVELLE
}

\section{Le neutrophile : une cellule choc}

\section{Un nouveau mécanisme du choc anaphylactique}

Friederike Jönsson, David A. Mancardi, Marc Daëron, Pierre Bruhns
Département d'immunologie, Inserm U760, Unité d'allergologie moléculaire et cellulaire, Institut Pasteur, 25, rue du Docteur Roux, 75015 Paris Cedex 15, France. bruhns@pasteur.fr
> L'anaphylaxie est une réaction allergique hyperaiguë pouvant être mortelle, dont l'incidence augmente en parallèle avec celle des maladies allergiques. Les anaphylaxies les plus fréquentes sont dues à des allergies alimentaires [1], médicamenteuses ou aux venins [2]. II s'agit d'une manifestation d'hypersensibilité immédiate due à la libération de médiateurs vasoactifs chez un sujet au préalable sensibilisé. Ces médiateurs induisent une vasodilatation des vaisseaux, une augmentation de la perméabilité vasculaire et une constriction bronchique qui concourent à induire une chute de la pression artérielle, des troubles respiratoires ainsi que la mort par arrêt circulatoire ou par un spasme majeur au niveau des bronches [3]. Le traitement du choc consiste en l'emploi d'antihistaminiques et de bronchodilatateurs, suivi, le cas échéant d'une injection intramusculaire d'adrénaline pour contrer la constriction bronchique et la vasodilatation des vaisseaux. Malgré ces traitements, le choc anaphylactique reste mortel dans certains cas.

\section{L'anaphylaxie : une histoire}

\section{uniquement d'IgE et de mastocytes ?}

Les conditions d'urgence dans lesquelles surviennent les chocs anaphylactiques limitent considérablement leur étude en clinique chez l'homme, et les mécanismes conduisant à l'induction du choc restent spéculatifs. Deux grands modèles d'anaphylaxie expérimentale chez l'animal ont été développés pour identifier des mécanismes. L'anaphylaxie active est induite en réponse à l'injection d'allergène chez des souris préalablement immunisées avec cet allergène. L'anaphylaxie passive est induite dans des souris naïves auxquelles on administre des anticorps spécifiques de l'allergène, puis une injection de l'allergène. Les modèles passifs ont permis de proposer les immunoglobulines $\varepsilon$ ( $\mid g \varepsilon$ ) et l'agrégation de leurs récepteurs de forte affinité FcERI à la surface des mastocytes comme voie d'induction du choc anaphylactique [4]. Des modèles passifs dans lesquels des immunoglobulines $G$ ( $\operatorname{lgG}$ ), et non plus des $\lg \varepsilon$, sont injectées ont ensuite suggéré que l'agrégation de récepteurs activateurs de faible affinité pour les IgG (FcyRIIIA) pouvait constituer une voie d'induction de l'anaphylaxie [5]. Les FcyRIIIA sont exprimés par de multiples cellules chez la souris, dont les mastocytes, basophiles et monocytes/ macrophages. Récemment, ce sont les basophiles qui ont été identifiés comme responsables du choc induit par les IgG chez la souris, grâce à l'emploi d'anticorps déplétant les basophiles [6]. Ce même travail a démontré que la déplétion des basophiles n'affectait pas le choc anaphylactique passif à $\lg \varepsilon$, qui, lui, est dépendant des mastocytes (ce que montre l'étude des souris déficientes en mastocytes, $W / W^{v}$ ou $\left.W^{\text {sh }}\right)$. En conclusion, dans les modèles d'anaphylaxie passive à $\lg \varepsilon$, l'activation des seuls mastocytes serait responsable du choc, par la libération de médiateurs dont l'histamine, et dans les modèles d'anaphylaxie passive à IgG, l'activation des seuls basophiles serait responsable du choc, par la libération de médiateurs dont le platelet activating factor (PAF) [7].

De façon surprenante, les résultats obtenus dans des modèles d'anaphylaxie passive ne corrèlent pas avec les modèles d'anaphylaxie active, qui sont plus proches de la physiologie du choc allergique chez l'homme. En effet, ni les Ig [8], ni les FcERI [9], ni les mastocytes [4], ni les basophiles ne sont nécessaires à l'induction du choc anaphylactique actif. Même l'absence de ces deux populations cellulaires en même temps n'abolit pas le choc anaphylactique actif [6]. D'autres anticorps que les $\lg \varepsilon$, et d'autres populations cellulaires que les mastocytes et les basophiles seraient donc capables, elles aussi, d'induire un choc anaphylactique.

\section{Le choc anaphylactique \\ « neutrophilique » dévoilé}

Notre étude publiée récemment dans The Journal of Clinical Investigation démontre que les neutrophiles, les récepteurs

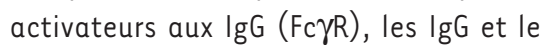
PAF jouent un rôle majeur dans l'anaphylaxie systémique active [10]. Ce modèle d'anaphylaxie est létal pour des souris sauvages, mais pas pour des souris n'exprimant pas de récepteurs activateurs pour les anticorps. En revanche, ni l'absence des FcERI, ni celle des FcyRIIIA, ni même l'absence cumulée des deux récepteurs pour les IgE (FcERI et FcERII) et de trois récepteurs pour les IgG (FcyRI,

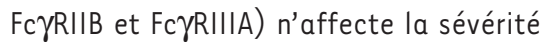
ou la mortalité induites par le choc. Ces 


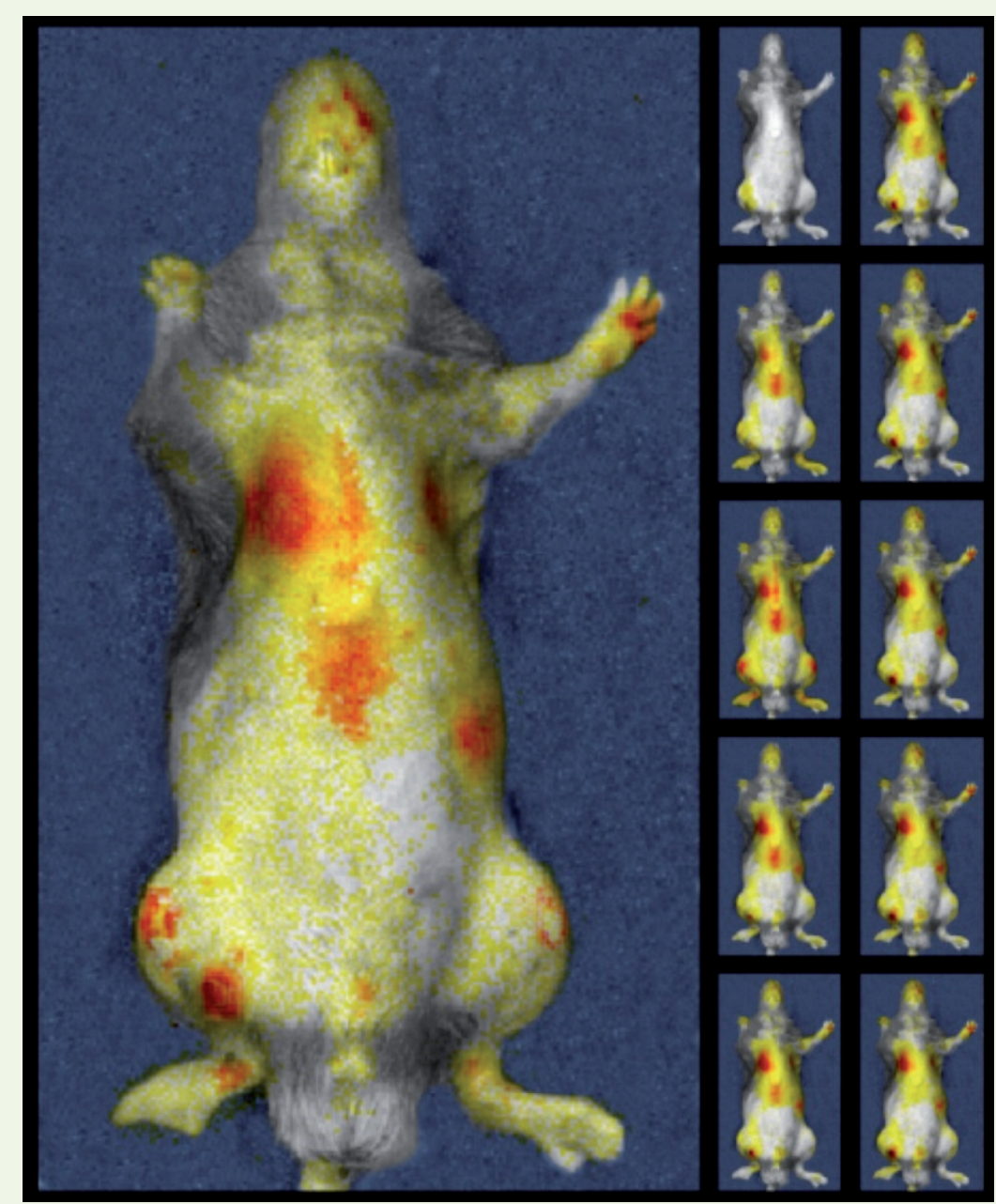

Figure 1. Cinétique d'activation de neutrophiles in vivo durant le choc anaphylactique actif. Des souris $5 K 0$ immunisées avec l'allergène ont été anesthésiées, injectées avec l'allergène par voie intraveineuse et $5 \mathrm{mn}$ plus tard avec du luminol par voie intrapéritonéale. Les images représentent l'accumulation de photons libérés par la dégradation du luminol pendant l'intégralité de la durée de l'expérience (grande image de gauche) ou à des intervalles successifs d'acquisition de photons de 2 min (cinétique) ordonnées de haut $(t=0)$ en bas, et de gauche à droite, sur un total de $20 \mathrm{~min}$ (petites images de droite). La quantité de photons, proportionnelle à l'activité de la MPO (myéloperoxydase) et donc à l'activation des neutrophiles in vivo, est représentée par un code couleur (du moins intense [jaune pâle] au plus intense [rouge vif] surimposé à une photographie en noir et blanc de la souris).

souris, déficientes pour 5 récepteurs aux IgE et IgG (souris $5 \mathrm{KO}$ ), expriment un seul récepteur activateur pour les IgG, le FcyRIV [11]. L'injection d'anticorps anti-Fc $\gamma$ RIV bloquants abolit le choc dans les souris 5KO. Le FcyRIV est exprimé uniquement par les monocytes/ macrophages et les neutrophiles qui ne sont pas, a priori, des effecteurs du choc allergique. Cependant, le choc anaphylactique est aboli chez les souris durant le choc anaphylactique in vivo par la technique de bioluminescence (Figure 1).

Nous avons cherché à savoir si, chez des souris sauvages aussi, les neutrophiles contribuent au choc anaphylactique actif. La déplétion de neutrophiles chez des souris sauvages inhibe fortement le choc. Les neutrophiles contribuent donc au choc anaphylactique actif chez les souris sauvages. Alors que la déplétion des basophiles n'affecte pas le choc, la déplétion cumulée de neutrophiles et de basophiles l'abolit chez des souris sauvages. Les basophiles contribuent donc aussi à cette réaction, mais de manière plus modeste. Finalement, notre étude démontre que le transfert de neutrophiles murins ou de neutrophiles humains restaure le choc anaphylactique chez des souris résistantes au choc anaphylactique actif, n'exprimant pas de FcR activateurs [10]. Les neutrophiles sont donc suffisants pour induire un choc anaphylactique actif.

\section{IgE ou IgG ? Histamine ou PAF ?}

L'utilisation de souris $5 K O$ a permis de démontrer la capacité du FcyRIV à déclencher un choc anaphylactique actif, mais ne démontre pas sa participation au choc dans des souris sauvages. Grâce à l'utilisation d'anticorps bloquants, nous avons démontré que ce sont deux récepteurs aux IgG, Fc $\gamma R$ IIIA et FcyRIV, qui sont responsables du choc dans des souris sauvages. Et pourtant, dans ces conditions où le choc est aboli, des IgE anti-allergène sont présentes et les FcERI sont exprimés et fonctionnels à la surface des mastocytes et des basophiles. Les IgG, et non les IgE, contribuent donc de manière prédominante au choc anaphylactique actif. Le FcyRIIIA est exprimé, comme le FcyRIV, à la surface des neutrophiles et des monocytes/macrophages, mais également à la surface des mastocytes, basophiles, et éosinophiles. Toutes ces cellules sont capables de libérer du PAF après activation [13], et les mastocytes et les basophiles également de l'histamine. L'utili- 
sation d'antagonistes démontre que le PAF, mais pas l'histamine, contribue de manière prépondérante au choc anaphylactique actif chez les souris sauvages. De plus, des souris déficientes en phospholipase-A2 et qui ne produisent pas de PAF [14], sont peu sensibles à I'induction du choc anaphylactique actif [10]. Le PAF a aussi été incriminé dans les manifestations anaphylactiques en clinique, et la concentration sérique de PAF corrélée avec la gravité du choc anaphylactique chez l'homme [15].

Ces résultats divergent du dogme [allergène + Ig + mastocytes $\rightarrow$ histamine = anaphylaxie] et proposent plutôt que les neutrophiles et les basophiles soient principalement à l'origine du choc, en libérant du PAF suite à l'agrégation de leurs récepteurs aux IgG, les Fc $\gamma R$ RIIIA et les FcyRIV. Les neutrophiles étant la population leucocytaire humaine majoritaire, et des IgG anti-allergène étant présents chez les allergiques, ce nouveau mécanisme de l'anaphylaxie, décrit pour l'instant uniquement chez la souris, pourrait contribuer au choc anaphylactique chez l'homme. Ce mécanisme « alternatif » dépendant des IgG et des neutrophiles pourrait être responsable de cas d'anaphylaxie indépendants des $\lg \varepsilon$, et même contribuer, avec les mécanismes «classiques » faisant intervenir des IgE et des mastocytes, à la gravité des chocs anaphylactiques. $\diamond$ An unexpected role for neutrophils in anaphylaxis

\section{CONFLIT D'INTÉRÊTS}

Les auteurs déclarent n'avoir aucun conflit d'intérêts concernant les données publiées dans cet article.

\section{REMERCIEMENTS}

Montage photo: M. J.M. Panaud, Image et reprographie, Institut Pasteur, Paris, France.

\section{RÉFÉRENCES}

1. Moneret-Vautrin DA. Facteurs de risque d'anaphylaxie alimentaire sévère : rôle confirmé de certaines classes de médicaments. Med Sci (Paris) 2010 ; $26: 719-23$.

2. Chippaux JP. Incidence mondiale et prise en charge des envenimations ophidiennes et scorpioniques. Med Sci (Paris) $2009 ; 25: 197-200$.

3. Galli SJ. Pathogenesis and management of anaphylaxis: current status and future challenges. J Allergy Clin Immunol 2005 ; 115 : 571-4.

4. Dombrowicz D, Flamand V, Brigman KK, et al. Abolition of anaphylaxis by targeted disruption of the high affinity immunoglobulin $\varepsilon$ receptor alpha chain gene. Cell $1993 ; 75: 969-76$.

5. Hazenbos WL, Gessner JE, Hofhuis FM, et al. Impaired IgG-dependent anaphylaxis and Arthus reaction in $\mathrm{Fc}$ gamma RIII (CD16) deficient mice. Immunity 1996 ; $5: 181-8$.
6. Tsujimura Y, Obata K, Mukai K, et al. Basophils play a pivotal role in immunoglobulin-G-mediated but not immunoglobulin- $\varepsilon$-mediated systemic anaphylaxis. Immunity 2008 ; 28 : 581-9.

7. Mukai K, Obata K, Tsujimura Y, Karasuyama H. New insights into the roles for basophils in acute and chronic allergy. Allergol Int 2009 ; 58 : 11-9.

8. Oettgen HC, Martin TR, Wynshaw-Boris A, et al. Active anaphylaxis in Ig\&-deficient mice. Nature $1994 ; 370$ : 367-70.

9. Miyajima I, Dombrowicz D, Martin TR, et al. Systemic anaphylaxis in the mouse can be mediated largely through IgGl and Fc gammaRIII. Assessment of the cardiopulmonary changes, mast cell degranulation, and death associated with active or IgE- or IgGldependent passive anaphylaxis. J Clin Invest 1997 ; 99: 901-14.

10. Jönsson F, Mancardi DA, Kita Y, et al. Mouse and human neutrophils induce anaphylaxis. J Clin Invest $2011 ; 121: 1484-96$.

11. Mancardi DA, lannascoli B, Hoos S, et al. FcgammaRIV is a mouse $\lg \varepsilon$ receptor that resembles macrophage FcepsilonRI in humans and promotes IgE-induced lung inflammation. J Clin Invest 2008 ; 118 : 3738-50.

12. Gross S, Gammon ST, Moss BL, et al. Bioluminescence imaging of myeloperoxidase activity in vivo. Nat Med $2009 ; 15: 455-61$.

13. Jouvin-Marche $\varepsilon$, Ninio $\varepsilon$, Beaurain G, et al. Biosynthesis of Paf-acether (platelet-activating factor). VII. Precursors of Paf-acether and acetyltransferase activity in human leukocytes. J Immunol $1984 ; 133: 892-8$.

14. Bonventre JV, Huang Z, Taheri MR, et al. Reduced fertility and postischaemic brain injury in mice deficient in cytosolic phospholipase A2. Nature 1997 ; $390: 622-5$.

15. Vadas $P$, Gold M, Perelman B, et al. Platelet-activating factor, PAF acetylhydrolase, and severe anaphylaxis. N Engl J Med 2008 ; 358 : 28-35.

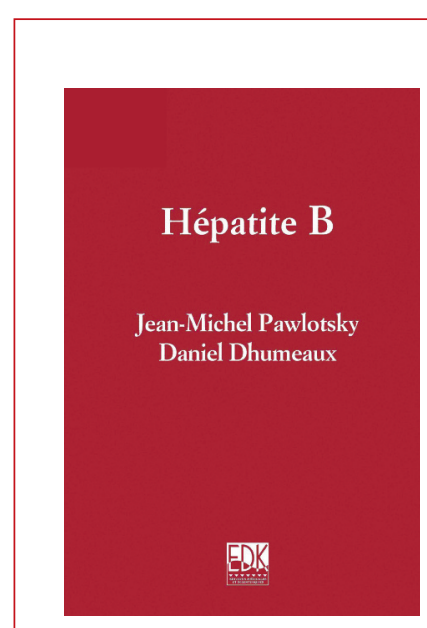

ISBN : 978-2-8425-4131-6 576 pages

\section{Bon de commande}

À retourner à EDK, 2, rue Troyon - 92316 Sèvres Cedex

Tél. : 0155641393 - Fax : 0155641394 - E-mail : edk@edk.fr

NOM : Prénom :

Adresse :

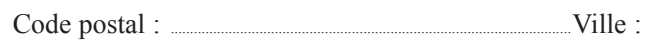

Pays :

Fonction :

Je souhaite recevoir l'ouvrage Hépatite B : $54 €+3 €$ de port $=\mathbf{5 7} €$ TTC offre exceptionnelle réservée aux abonnés à $\mathrm{m} / \mathrm{s}$ jusqu'au 31 décembre 2010

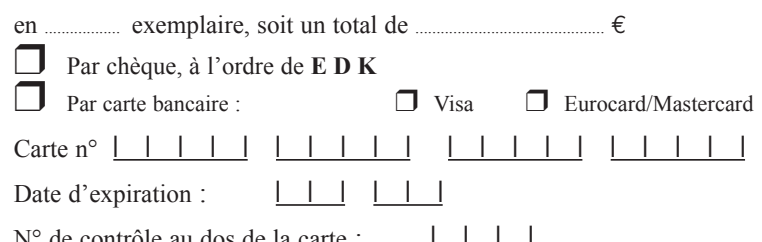

Signature :

$\mathrm{N}^{\circ}$ de contrôle au dos de la carte : $\quad$ l $\quad$ | $\quad$ | | 